\title{
Local amplitude and phase retrieval method for digital holography applied to microscopy
}

\author{
Michael Liebling*, Thierry Blu, Etienne Cuche, \\ Pierre Marquet ${ }^{\dagger}$, Christian Depeursinge, Michael Unser \\ BIO-E, STI, École Polytechnique Fédérale de Lausanne, CH-1015 Lausanne, Switzerland \\ ${ }^{\dagger}$ Institut de Physiologie, Université de Lausanne, CH-1005 Lausanne, Switzerland
}

\begin{abstract}
We present a numerical two-step reconstruction procedure for digital off-axis Fresnel holograms. First, we retrieve the amplitude and phase of the object wave in the CCD plane. For each point we solve a weighted linear set of equations in the least-squares sense. The algorithm has $O(N)$ complexity and gives great flexibility. Second, we numerically propagate the obtained wave to achieve proper focus. We apply the method to microscopy and demonstrate its suitability for the real time imaging of biological samples.
\end{abstract}

Keywords: Digital holography, Fresnel transform, Fresnelets, Least-squares, Phase retrieval.

\section{INTRODUCTION}

Digital holography was first proposed, some twenty years after Gabor's invention of holography, ${ }^{1}$ by Goodman et al. ${ }^{2}$ and Kronrod et al. ${ }^{3}$ Since then, it has constantly evolved, allowing the acquisitions to be done using a CCD camera ${ }^{4}$ and the phase to be reconstructed in addition to the sole amplitude. ${ }^{5}$ A promising perspective is its application to microscopy, which allows for truly non-invasive examinations of biological samples. Indeed, the technique has the ability to detect sub-wavelength changes in the morphology of living organisms. ${ }^{6}$

Most digital holography processing methods that have been proposed so far mimic the physical reconstruction process by which the hologram plate is illuminated with the same source used to record it, such that the diffracted wave yields an image (resp. virtual image). The implementation basically consists in simulating this process on digitally acquired holograms. This is usually achieved by computing the Fresnel transform of the hologram which may have been multiplied by the appropriate digital counterpart of a reference wave beforehand. The main drawback of this approach is that the reconstructed image is corrupted by interference terms, the zero-order and twin-image. This is usually dealt with by applying a suitable filter to remove them.

Here, we propose a two step algorithm for reconstructing digital holograms. First, we retrieve the amplitude and phase of the object wave in the CCD plane by solving a weighted linear set of equations in the least-squares sense. Second, we numerically propagate the obtained wave to achieve proper focus. The advantage is that the zero-order and twin-image are (intrinsically) removed during the phase retrieval step.

The paper is organized as follows: in Section 2, we develop the phase retrieval algorithm. In Section 3, we describe the experimental methods. Finally, in Section 4, we present the experimental results carried out on biological samples.

\section{PHASE RETRIEVAL ALGORITHM}

The hologram, measured by the CCD camera, $I(\mathbf{x}) \in \mathbb{R}_{+}$results from the interference, at every location $\mathbf{x}=(x, y)$, of the object wave $\Psi(\mathbf{x}) \in \mathbb{C}$ with a reference wave $R(\mathbf{x})=A(\mathbf{x}) \exp (i \theta(\mathbf{x}))$ where $A(\mathbf{x}) \in \mathbb{R}_{+}, \theta(\mathbf{x}) \in \mathbb{R}$

$$
I(\mathbf{x})=|\Psi(\mathbf{x})+R(\mathbf{x})|^{2}
$$

The task is to recover $\Psi(\mathbf{x})$, which is complex, from measurements of $I(\mathbf{x})$ that are real. The difficulty is that at each measurement location $\mathbf{x}$, we only have one equation but two unknowns (the real and imaginary part of $\Psi(\mathbf{x})$ ), assuming

*E-mail: michael.liebling AT epfl.ch, Telephone: +41 21693 51 43, Fax.: +41 216933701.

Copyright (C) 2003 Society of Photo-Optical Instrumentation Engineers.

This paper will be published in the proceedings of the European Conference on Biomedical Optics 2003, $22-25$ June 2003, Munich, Germany and is made available as an electronic preprint with permission of SPIE. One print or electronic copy may be made for personal use only. Systematic or multiple reproduction, distribution to multiple locations via electronic or other means, duplication of any material in this paper for a fee or for commercial purposes, or modification of the content of the paper are prohibited. 


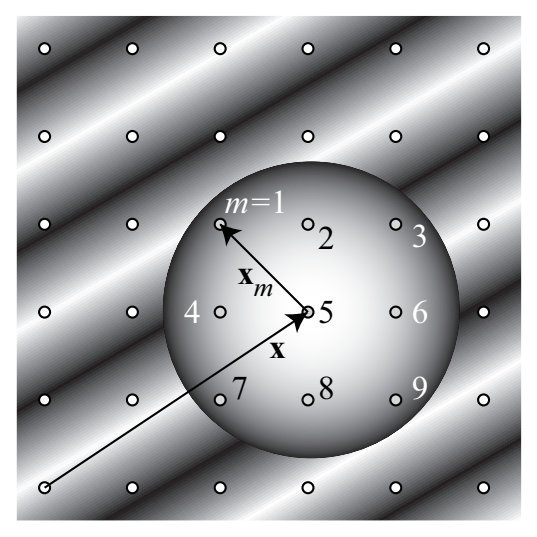

Figure 1. Schematic hologram with the weighting function overlaid at position $\mathbf{x}$. The latter has a Gaussian shaped aspect: a lower weight is given to points that are far away from the point of interest. The dots denote the pixels' centers.

that the reference wave $R(\mathbf{x})$ is known. Furthermore, although the reference's phase $\theta(\mathbf{x})$ can be modeled with good accuracy (we assume it is known), this is not the case for its amplitude $A(\mathbf{x})$. Therefore, we do not try to model the latter, which becomes an additional unknown.

The central point in our algorithm is that we consider that the unknown quantities $\Psi(\mathbf{x})$ and $A(\mathbf{x})$ vary slowly with the spatial variable $\mathbf{x}$. Conversely, we assume that $I(\mathbf{x})$ and $\theta(\mathbf{x})$ vary more rapidly. With these hypotheses, we can determine the phase and amplitude of $\Psi(\mathbf{x})$ together with $A(\mathbf{x})$ by solving, at each location $\mathbf{x}$, the set of $M$ non-linear equations

$$
I\left(\mathbf{x}+\mathbf{x}_{m}\right)=\left|\Psi(\mathbf{x})+A(\mathbf{x}) \exp \left(i \theta\left(\mathbf{x}+\mathbf{x}_{m}\right)\right)\right|^{2}
$$

where the $\mathbf{x}+\mathbf{x}_{m}(m=1, \ldots, M)$ are the locations of the $M$ pixels within the considered neighborhood of $\mathbf{x}$ (see Fig.1, where $M=9$ ). By using a more concise notation we rewrite this relation as

$$
I_{m}=\left|\Psi+A \exp \left(i \theta_{m}\right)\right|^{2}=|\Psi|^{2}+A^{2}+2 \Re\left(R_{m}^{*} \Psi\right)
$$

We aim at finding a solution in the least-squares sense, viz.

$$
\arg \min _{A \in \mathbb{R}_{+}^{*}, \Psi \in \mathbb{C}} \sum_{m} w_{m}\left|I_{m}-\left(|\Psi|^{2}+A^{2}+2 \Re\left(R_{m}^{*} \Psi\right)\right)\right|^{2} .
$$

The weights $w_{m}$ ensure that points that are far away from the pixel of interest account for less than those that are in its close vicinity. We have set the weights according to a Gaussian shaped weighting function centered over the pixel of interest (Fig.1). In order to carry out the computations, we convert equation (4) to a linear problem, by performing the change of variables $\Phi=A \Psi, U=A^{2}+|\Phi|^{2} / A^{2},(\Phi \in \mathbb{C}$ and $U \in \mathbb{R}), V_{m}=\exp \left(i \theta_{m}\right)$

$$
\arg \min _{U, \Phi} \sum_{m} w_{m}\left|I_{m}-U-2 \mathfrak{R}\left(V_{m} \Phi\right)\right|^{2} .
$$

Consequently, $U$ and $\Phi$, must be the solutions of the set of normal equations

$$
\left\{\begin{array}{l}
\sum_{m} w_{m}\left(I_{m}-U-2 \Re\left(V_{m} \Phi\right)\right)=0 \\
\sum_{m} w_{m} V_{m}\left(I_{m}-U-2 \Re\left(V_{m} \Phi\right)\right)=0 \\
\sum_{m} w_{m} V_{m}^{*}\left(I_{m}-U-2 \Re\left(V_{m} \Phi\right)\right)=0
\end{array}\right.
$$

obtained by differentiating the expression in (5) with respect to $U, \Phi$ and $\Phi^{*}$. If we normalize the $w_{m}$ such that $\sum_{m} w_{m}=1$ and rearrange the terms, we get

$$
\left\{\begin{array}{l}
\sum_{m} w_{m} I_{m}=U+2 \Re\left(\Phi \sum_{m} w_{m} V_{m}\right) \\
\sum_{m} w_{m} V_{m} I_{m}=U \sum_{m} w_{m} V_{m}+\left(\sum_{m} w_{m} V_{m}^{2}\right) \Phi+\Phi^{*} \\
\sum_{m} w_{m} V_{m}^{*} I_{m}=U \sum_{m} w_{m} V_{m}^{*}+\Phi+\left(\sum_{m} w_{m} V_{m}^{* 2}\right) \Phi^{*}
\end{array}\right.
$$


If we let $\alpha=\sum_{m} w_{m} V_{m}$ and $\beta=\sum_{m} w_{m} V_{m}^{2}$, we finally end up with the linear system of equations

$$
\left(\begin{array}{lll}
1 & \alpha & \alpha^{*} \\
\alpha & \beta & 1 \\
\alpha^{*} & 1 & \beta^{*}
\end{array}\right)\left(\begin{array}{l}
U \\
\Phi \\
\Phi^{*}
\end{array}\right)=\left(\begin{array}{l}
\sum_{m} w_{m} I_{m} \\
\sum_{m} w_{m} V_{m} I_{m} \\
\sum_{m} w_{m} V_{m}^{*} I_{m}
\end{array}\right)
$$

that is to be solved for each pixel. The only remaining point is to model the reference wave's phase $\theta(\mathbf{x})$, which can be done with good accuracy if the reference wave is plane or has a quadratic phase. Finally, we restore the original variables $\Psi$ and $A$ by the non-linear operations

$$
\begin{aligned}
& A=\left(\frac{U+\sqrt{U^{2}-4|\Phi|^{2}}}{2}\right)^{1 / 2} \\
& \Psi=\frac{\Phi}{A} .
\end{aligned}
$$

Since the algorithm is local, it has a complexity $O(N)$, where $N$ is the number of pixels in the image.

\section{METHODS}

The holograms are acquired using a digital holographic microscopy setup for transmission imaging of transparent samples, as described elsewhere. ${ }^{6}$ The specimen is illuminated by a plane wave and the transmitted light is collected by a microscope objective. The latter produces a wavefront - the object wave-whose interference with a reference wave is recorded on a CCD camera in an off-axis geometry. The complex object wave $\Psi_{\mathrm{CCD}}(\mathbf{x})$ in the CCD plane can be modeled by

$$
\Psi_{\mathrm{CCD}}(\mathbf{x})=\Psi(\mathbf{x}) \exp \left(i\|\mathbf{x}\|^{2} / D\right), D \in \mathbb{R}^{*}
$$

where $\Psi(\mathbf{x})$ is a magnified version of the object wave in the vicinity of the sample; the quadratic phase term is a consequence of the wave's travel through the microscope objective. Thus the measured interference with a plane wave (whose wave vector is $\left.\mathbf{k}=\left(k_{x}, k_{y}, k_{z}\right)\right)$ in the CCD plane is

$$
I(\mathbf{x})=\left|A(\mathbf{x}) \exp \left(i\left(k_{x} x+k_{y} y\right)\right)+\Psi(\mathbf{x}) \exp \left(i\|\mathbf{x}\|^{2} / D\right)\right|^{2} .
$$

The latter equation may be written equivalently as

$$
I(\mathbf{x})=\left|A(\mathbf{x}) \exp \left(i\left(1 / D^{\prime}\left\|\mathbf{x}-\mathbf{x}_{o}\right\|^{2}+C\right)\right)+\Psi(\mathbf{x})\right|^{2}
$$

where $\mathbf{x}_{o}=\left(x_{o}, y_{o}\right) \in \mathbb{R}^{2}, C \in \mathbb{R}, D^{\prime} \in \mathbb{R}^{*}$, and interpreted as the interference of the complex wave $\Psi$ with a non-planar wave. It is thus sufficient to adjust the parameters $D^{\prime}, x_{o}$ and $y_{o}$ appropriately to specify our model $\theta(\mathbf{x})$. In the forthcoming experiment, this calibration step was done manually.

When the CCD is not in the image plane, but at a distance $d$, the complex wave that we reconstruct from the hologram is out of focus. The defocus must then be corrected by numerically propagating the reconstructed wavefront to the proper distance and achieve a sharp image, for example using the numerical Fresnelet transform. ${ }^{7}$

\section{RESULTS}

We have applied the above proposed technique to the reconstruction of images acquired in the framework of a functional morphology experiment on living cells. The system was used to track changes in their morphology in response to a variation of the perfusion liquid's concentration. This time phenomenon can be observed at video rate since off-axis holography only requires one data acquisition frame per reconstructed image.

The hologram is shown in Fig.2(a). The reconstruction from the same data set using an alternative technique ${ }^{6}$ (but without applying any zero-order or twin-image removal scheme) is displayed in Fig. 2(b). In this approach, the quadraticphase exponential induced by the objective is compensated numerically by multiplying the diffracted wave by a numerical phase mask. The zero-order is the square portion that masks the center of the image. The image itself is on the upper right while the twin-image is located in the lower left. Only the image is in focus. 


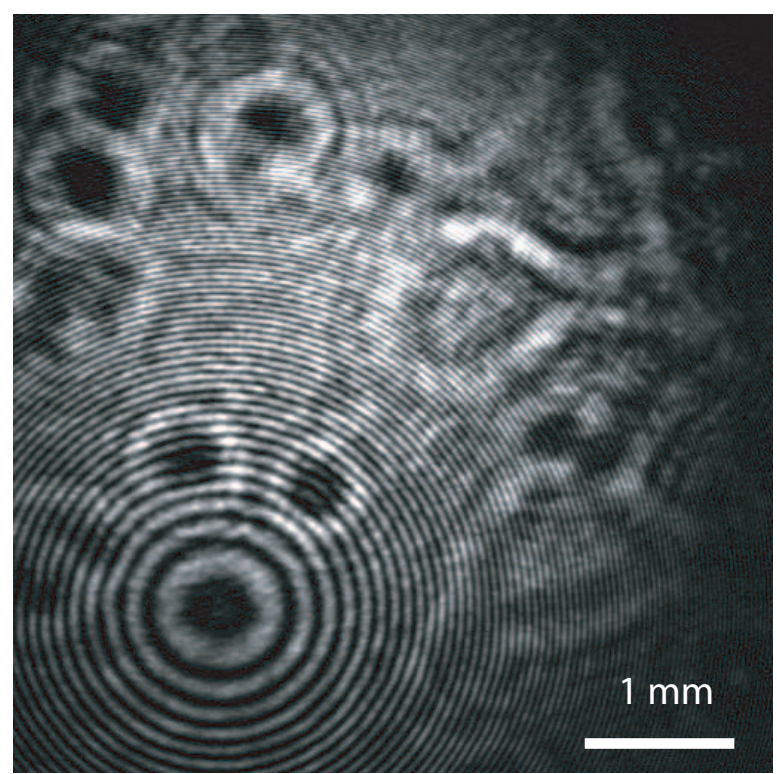

(a)

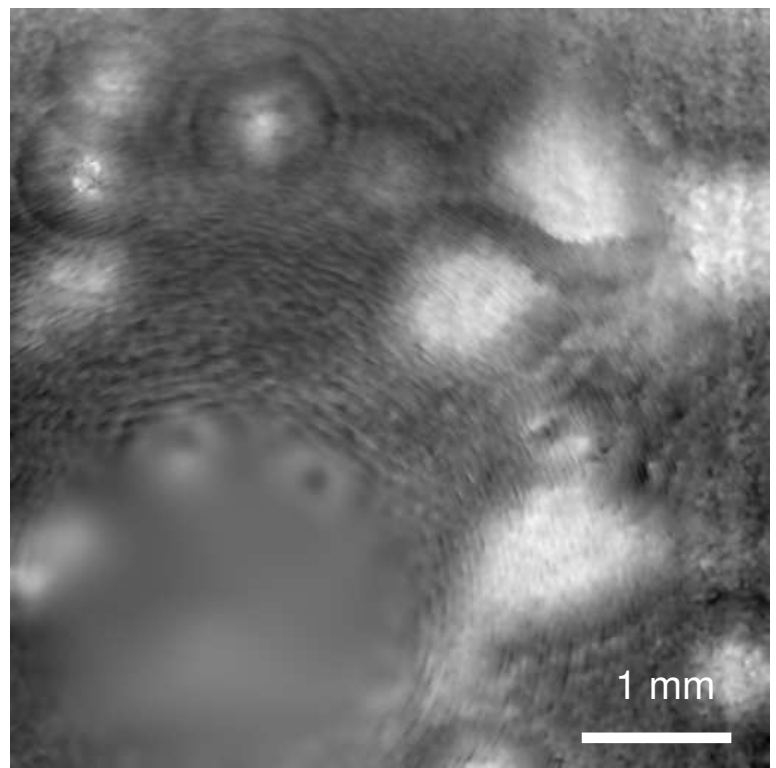

(c)

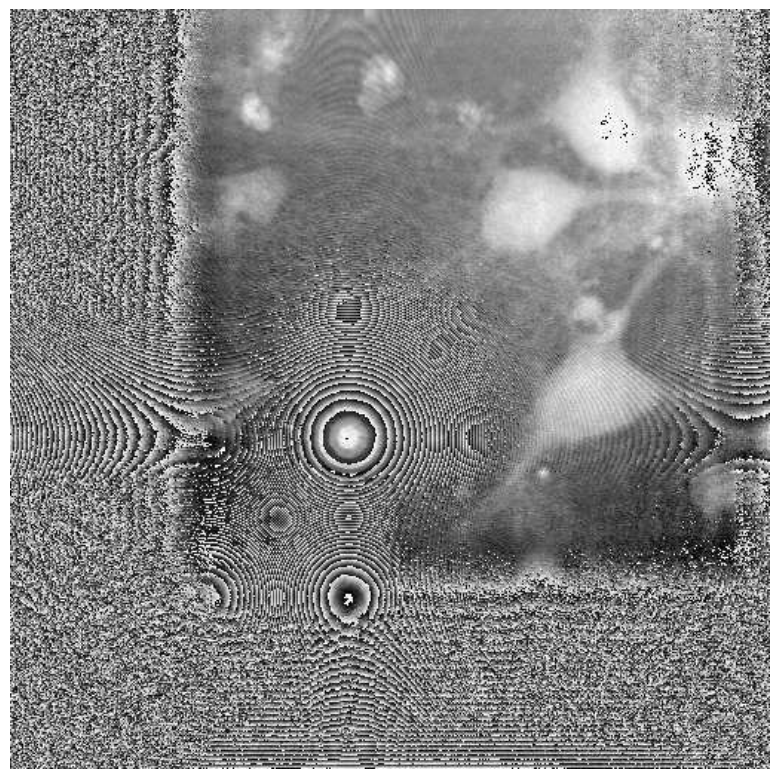

(b)

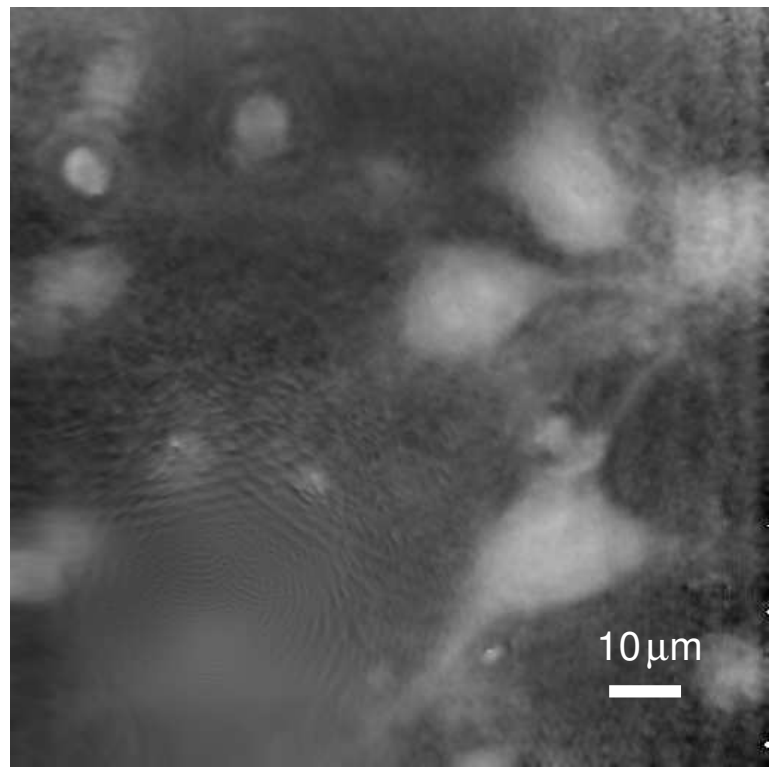

(d)

Figure 2. (a) Measured hologram, (b) Reconstructed phase using a method as described in Cuche et al., ${ }^{6}$ (c) Reconstructed phase in the CCD plane, (d) Reconstructed phase, with adjusted focus. All images are $512 \times 512$ pixels.

In Fig. 2(c), we show the phase in the CCD plane (i.e. $\arg \Psi$ ) that was recovered from our phase retrieval algorithm. The bodies of the cells are already recognizable (white blobs) but the image is blurred since the CCD camera is not in the image plane. The recovered wave front is then propagated numerically to the image plane at the proper distance (adjusted to $d=10.1 \mathrm{~cm}$, for $\lambda=632.8 \mathrm{~nm}$ ); the final reconstructed image is shown in Fig. 2(d).

Remarkably, the reconstructions using the new approach are not perturbed by the zero-order terms which results in a larger field of view. 


\section{CONCLUSION}

We have presented a digital hologram reconstruction method. The applicability of the technique to real world images from biology was also demonstrated. The phase and amplitude of the object wave are first reconstructed in the CCD plane. The object wave is then numerically propagated to be in proper focus. This approach has many advantages. It offers more flexibility since the various problems (phase retrieval, wave propagation) are decoupled. This makes the technique applicable over a wider range of experimental conditions. Also, because the method is local—compared to methods that operate in the Fourier domain-it will be less sensitive to local defects in the measurements and will not deteriorate the quality over the whole reconstruction area. Furthermore, since the reconstructions do not contain the zero-order and twinimage terms that usually limit the field of view, there is no waste of data. We therefore take full advantage of the off-axis geometry which requires one single acquisition per phase reconstruction, enabling measurements at video rate. This makes this technique very relevant for applications in biology.

\section{REFERENCES}

1. D. Gabor, “A new microscopic principle,” Nature 161, pp. 777-778, May 1948.

2. J. W. Goodman and R. W. Lawrence, "Digital image formation from electronically detected holograms," Appl. Phys. Lett. 11, pp. 77-79, Aug. 1967.

3. M. A. Kronrod, N. S. Merzlyakov, and L. Yaroslavskii, "Reconstruction of a hologram with a computer," Sov. Phys. Tech. Phys. 17, pp. 333-334, Aug. 1972.

4. U. Schnars and W. Jüptner, "Direct recording of holograms by a CCD target and numerical reconstruction," Appl. Opt. 33, pp. 179-181, Jan. 1994.

5. E. Cuche, F. Bevilacqua, and C. Depeursinge, "Digital holography for quantitative phase-contrast imaging," Opt. Lett. 24, pp. 291-293, Mar. 1999.

6. E. Cuche, P. Marquet, and C. Depeursinge, "Simultaneous amplitude-contrast and quantitative phase-contrast microscopy by numerical reconstruction of Fresnel off-axis holograms," Appl. Opt. 38, pp. 6994-7001, Dec. 1999.

7. M. Liebling, T. Blu, and M. Unser, "Fresnelets: New multiresolution wavelet bases for digital holography," IEEE Trans. Image Proc. 12, pp. 29-43, Jan. 2003. 